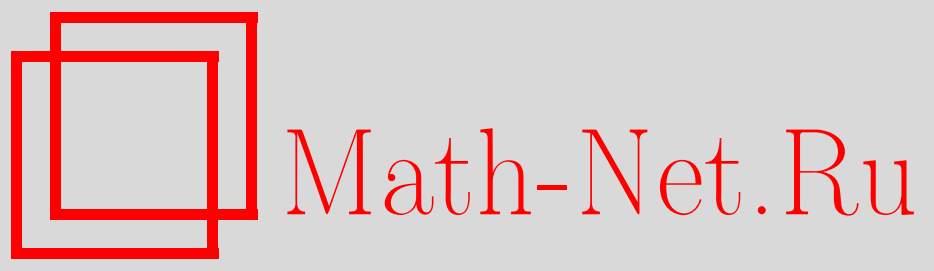

В. Н. Бородихин, Фазовые переходы и особенности роста зародышей новой фазы вещества, ТМФ, 2013, том 177, номер 1, 126-136

DOI: https://doi.org/10.4213/tmf8449

Использование Общероссийского математического портала Math-Net.Ru подразумевает, что вы прочитали и согласны с пользовательским соглашением http://www.mathnet.ru/rus/agreement

Параметры загрузки:

IP: 54.162 .127 .20

26 апреля 2023 г., 16:00:37

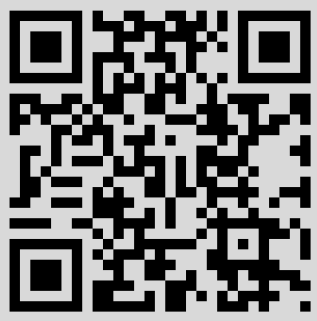




\section{ФАЗОВЫЕ ПЕРЕХОДЫ И ОСОБЕННОСТИ РОСТА ЗАРОДЫШЕЙ НОВОЙ ФАЗЫ ВЕЩЕСТВА}

Выполнено описание фазовых переходов с помощью одномерного дробнодифференциального кинетического уравнения типа Фоккера-Планка. Найдено общее решение, описывающее рост зародышей при фазовых переходах во фрактальной среде.

Ключевые слова: фазовые переходы дробного порядка, рост зародышей, дробно-дифференциальное уравнение Фоккера-Планка.

DOI: $1010.4231 / \operatorname{tmf} 8449$

\section{1. ВВЕДЕНИЕ}

Известно, что кинетика фазовых переходов может быть изучена с помощью уравнения Фоккера-Планка, в частности оно описывает рост зародышей новой фазы вещества, возникающих при фазовых переходах первого рода [1], [2].

Представляет интерес применение дробно-дифференциального обобщения кинетического уравнения для описания фазовых переходов, оно позволит учитывать некоторые дополнительные эффекты, которые могут происходить во фрактальных средах. В частности, это позволит исследовать эффекты нелокальности, памяти предыдущих состояний системы и некоторые другие эффекты [3].

Появление новых особенностей может быть связано с проявлением фрактальности сред. Фрактальные структуры могут являться следствием многих необратимых процессов и явлений, таких, например, как ударно-волновые явления в конденсированных средах [4], диффузия, агрегирование, разрушение, динамический хаос, растворение, турбулетное движение таких сред, как плазма, жидкие металлы, соленая вода и др. [3], [5].

Еще можно упомянуть системы, обладающие топологией перколирующего фрактального множества, а именно взаимодействие полей и токов в дальнем хвосте магнитосферы [6], процессы самоорганизации в низкотемпературной и замагниченной плазме [7], формирование фрактальной "паутины" из проводящих элементов в самоорганизующихся органических полимерах [8]. В данных системах возникают дальнодействующие корреляционные эффекты, проявляющиеся в негауссовом поведении кинетических процессов вблизи неравновесных квазистационарных состояний.

* Омский государственный университет, Омск, Россия. E-mail: borodikhin@inbox.ru 
Кроме того, в таких системах могут возникать эффекты неаддитивности и самоорганизуемой критичности [9].

\section{2. ДРОБНО-ДИФФЕРЕНЦИАЛЬНОЕ КИНЕТИЧЕСКОЕ УРАВНЕНИЕ}

В работе [10] получено дробно-дифференциальное обобщение одномерного кинетического уравнения Фоккера-Планка

$$
\frac{\partial^{\gamma} f}{\partial t^{\gamma}}=\frac{\partial^{\alpha}}{\partial(-x)^{\alpha}}(A f)+\frac{\partial^{\alpha_{1}}}{\partial(-x)^{\alpha_{1}}}(B f),
$$

где $A$ - функция скорости дрейфа, $B$ - диффузная функция, $f$ - функция распределения, $\partial^{\alpha} / \partial(-x)^{\alpha}$ - сумма правой и левой дробных производных Лиувилля, $0<\alpha<2, \alpha<\alpha_{1}<2$.

В случае $\alpha=1, \alpha_{1}=2, \gamma=1$ данное уравнение переходит в обычное кинетическое уравнение Фоккера-Планка

$$
\frac{\partial f}{\partial t}=-\frac{\partial}{\partial x}(A f)+\frac{1}{2} \frac{\partial^{2}}{\partial x^{2}}(B f) .
$$

Функция распределения $f(t, a)$, если рассматривать ее как распределение зерен новой фазы вещества по размерам в теории фазовых переходов, обычно нормируется таким образом, что

$$
N(t)=\int_{0}^{\infty} f(t, a) d a
$$

где $N(t)$ - число зерен в единице объема.

Перепишем уравнение (1), используя для удобства записи начальных условий в левой части дробную производную Капуто [11]

$$
{ }^{c} D_{t}^{\gamma} f(t)=\frac{1}{\Gamma(\alpha-n)} \int_{p}^{t} \frac{f^{(n)}(\tau) d \tau}{(t-\tau)^{\gamma+1-n}}, \quad n-1<\gamma<n,
$$

где $f^{(n)}$ - соответствующая производная целого порядка, $\Gamma$ - гамма-функция, а в правой части мы будем использовать производную Римана-Лиувилля

$$
D_{b+}^{\alpha} f(a)=\frac{1}{\Gamma(1-\alpha)} \frac{d}{d a} \int_{b}^{a} \frac{f(z) d z}{(a-z)^{\alpha}}, \quad 0 \leqslant \alpha \leqslant 1 .
$$

В итоге получим уравнение

$$
{ }^{c} D_{t}^{\gamma} f(t, a)=D_{b+}^{\alpha}[A(a) f(t, a)]+D_{b+}^{\alpha_{1}}[B(a) f(t, a)] .
$$

Поскольку производные Капуто и Римана-Лиувилля совпадают в пределе целого порядка, вид уравнения при $\gamma=1$ совпадает с уравнением (2).

Используя обобщенное правило Лейбница для дробной производной от произведения функций [12], перепишем уравнение (6) в виде

$$
{ }^{c} D_{t}^{\gamma} f(t, a)=\tilde{A}^{\alpha}(a) f(t, a)+\tilde{A}^{\alpha-1}(a) \frac{d f(t, a)}{d a}+\widetilde{B}^{\alpha_{1}}(a) f(t, a)+\widetilde{B}^{\alpha_{1}-1}(a) \frac{d f(t, a)}{d a},
$$


где

$$
\begin{aligned}
\tilde{A}^{\alpha}(a) & =\frac{\Gamma(\alpha+1)}{\Gamma(\alpha-1)} D_{b+}^{\alpha} A(a), & \tilde{A}^{\alpha-1}(a) & =\frac{\Gamma(\alpha+1)}{\Gamma(\alpha)} D_{b+}^{\alpha-1} A(a), \\
\widetilde{B}^{\alpha_{1}}(a) & =\frac{\Gamma\left(\alpha_{1}+1\right)}{\Gamma\left(\alpha_{1}-1\right)} D_{b+}^{\alpha_{1}} B(a), & \widetilde{B}^{\alpha_{1}-1}(a) & =\frac{\Gamma\left(\alpha_{1}+1\right)}{\Gamma\left(\alpha_{1}\right)} D_{b+}^{\alpha_{1}-1} B(a) .
\end{aligned}
$$

Общее решение будем искать в виде

$$
f(t, a)=f_{1}(t) f_{2}(a)
$$

Подставим (8) в (7) и поделим на (8), получим

$$
\frac{1}{f_{1}(t)}{ }^{c} D_{t}^{\gamma} f_{1}(t)=\tilde{A}^{\alpha}(a)+\widetilde{B}^{\alpha_{1}}(a)+\left(\tilde{A}^{\alpha-1}(a)+\widetilde{B}^{\alpha_{1}-1}(a)\right) \frac{1}{f_{2}(a)} \frac{d f_{2}(a)}{d a}=-\lambda .
$$

Таким образом, приходим к системе двух уравнений для функций $f_{1}(t)$ и $f_{2}(a)$ с разделяющимися переменными. Производную Капуто можно записать в виде дробного дифференциала [3]:

$$
\begin{aligned}
\frac{d^{\gamma} f_{1}}{f_{1}} & =-\lambda d t^{\gamma} \\
\frac{d f_{2}}{f_{2}} & =\frac{-\lambda-\tilde{A}^{\alpha}(a)-\widetilde{B}^{\alpha_{1}}(a)}{\tilde{A}^{\alpha-1}(a)+\widetilde{B}^{\alpha_{1}-1}(a)} d a .
\end{aligned}
$$

Общее решение есть произведение решений уравнений (10) и (11). В итоге получим

$$
f(t, a)=C t^{\gamma-1} E_{\gamma, \gamma}\left(-\lambda\left(t-t_{0}\right)^{\gamma}\right) e^{\varphi(a)},
$$

где

$$
\varphi(a)=\int \frac{-\lambda-\tilde{A}^{\alpha}(a)-\widetilde{B}^{\alpha_{1}}(a)}{\tilde{A}^{\alpha-1}(a)+\widetilde{B}^{\alpha_{1}-1}(a)} d a,
$$

$E_{\gamma, \gamma}$ - обобщенная функция Миттаг-Леффлера [13], являющаяся решением (10), $C, \lambda$ - соответственно константа интегрирования и собственное значение уравнения, определяемые из начальных и граничных условий.

На рис. 1 показаны графики зависимости решения (12) $f(t)$ от времени $(\lambda=1$, $\left.C=1, t_{0}=0, e^{\varphi(a)}=1\right)$ для трех значений $\gamma=1,3 / 4,1 / 4$.

Видно, что чем меньше $\gamma$, тем более резко при малых $t$ спадает функция. Из-за множителя $1 / t^{1-\gamma}$ функция в нуле обращается в бесконечность. Графики при $\gamma=$ $1 / 4,3 / 4$ показаны от $t=0.01$. При $\gamma=1$ обобщенная функция Миттаг-Леффлера переходит в экспоненциальную функцию, множитель $1 / t^{1-\gamma}$ становится равным единице и в нуле функция принимает конечное значение. Графики зависимости решения от $a$ приведены в следующем разделе.

\section{3. РОСТ ЗАРОДЫШЕЙ ПРИ ФАЗОВЫХ ПЕРЕХОДАХ}

Рост зародышей при фазовых переходах первого рода, в частности на начальной стадии, описывается уравнением Фоккера-Планка [1], [2]. Вместо термодинамической вероятности образования зародыша можно говорить о пропорциональной ей 


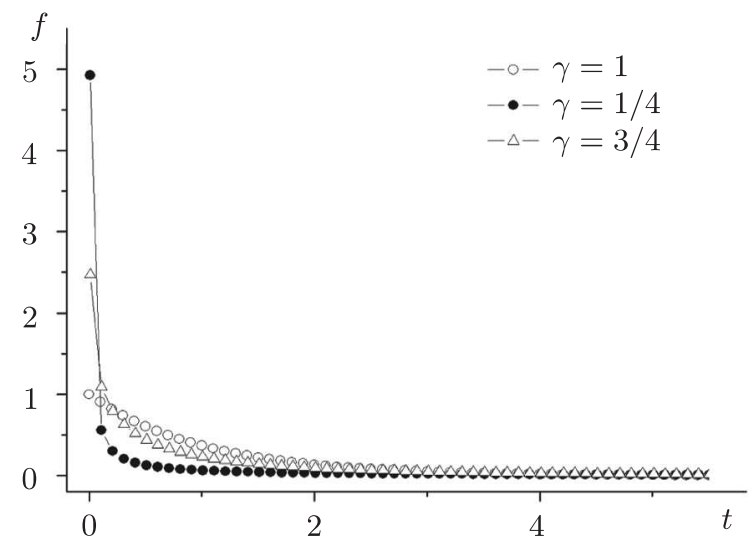

Рис. 1. Графики зависимости от времени функции (12) при различных $\gamma$.

равновесной функции распределения существующих в среде зародышей по размеpam

$$
f_{0}(a) \sim e^{-R_{\min } / T}
$$

где $R_{\min }$ - минимальная работа, которую необходимо затратить для образования зародыша заданного размера, $T$ - температура. Соответствующее термодинамическое равновесие относится к временам, малым по сравнению со временем образования критических зародышей, за которым следует переход в новую фазу.

Элементарным актом, изменяющим размер зародыша, является присоединение или потеря одной молекулы. Данное изменение следует считать малым, поскольку зародыши в излагаемой теории являются микроскопическими образованиями. Размер зародыша $a<a_{\mathrm{c}}$, где $a_{\mathrm{c}}-$ критический размер зародыша, соответственно зародыши бо́льших размеров развиваются в новую фазу вещества. Таблица значений размеров критических зародышей для воды в зависимости от давления приведена, например, в работе [1], они варьируются от $1.6 \cdot 10^{-5}$ м до $1.6 \cdot 10^{-10}$ м.

Таким образом, мы можем описывать рост зародышей в пространстве размеров кинетическим уравнением Фоккера-Планка

$$
\frac{\partial f}{\partial t}=-\frac{\partial s}{\partial a}
$$

где $s=A f-B(\partial f / \partial a)-$ плотность потока в пространстве размеров. Коэффициенты $A$ и $B$ связаны соотношением, следующим из условия обращения величины $s$ в нуль для равновесного распределения [2]. Таким образом, $A=-\left(B / f_{0}\right)\left(\partial f_{0} / \partial a\right)$ или, что эквивалентно, $A=-(B / T) R_{\min }^{\prime}$.

Для фазовых переходов равновесная функция $f_{0}(a)$ имеет вид распределения Гaycca $\left(a<a_{\mathrm{c}}\right)[2]$ :

$$
f_{0}(a)=f_{0}\left(a_{\mathrm{c}}\right) \exp \left(\frac{4 \pi \sigma}{k_{\mathrm{B}} T}\left(a-a_{\mathrm{c}}\right)^{2}\right),
$$

где $\sigma$ - коэффициент поверхностного натяжения,

$$
f_{0}\left(a_{\mathrm{c}}\right)=C_{0} \exp \left(\frac{-4 \pi \sigma a_{\mathrm{c}}^{2}}{3 k_{\mathrm{B}} T}\right)
$$

5 Теоретическая и математическая физика, т. 177, № 1, 2013 г. 
$C_{0}$ - некая константа, $k_{\mathrm{B}}$ - постоянная Больцмана. Функцию $f_{0}$ в виде $(15)$ можно использовать только при условии $a<a_{\mathrm{c}}$.

Распределение (15) может быть получено из (12) следующим образом. Коэффициент $A(a)$ выберем в виде $a_{\mathrm{c}} A_{1} A_{2} /\left(A_{1} a_{\mathrm{c}} a-A_{2} a^{2}\right)$, где $A_{1}, A_{2}$ - константы, коэффициентом $B(a)$ пренебрежем, что соответствует обратимым процессам. Равновесная функция $f_{0}$ применима только на стадии до возникновения фазового перехода, где данное приближение является допустимым на микроскопическом уровне. На стадии фазовых переходов необходимо учитывать коэффициент диффузии. После интегрирования получим $\varphi(a)=\lambda a^{3} /\left(3 A_{1} a_{\mathrm{c}}\right)-\lambda a^{2} /\left(2 A_{2}\right)$, здесь мы пренебрегли более медленной логарифмической зависимостью от $a$. Сопоставляя полученное выражение с (13) и работой, которую необходимо затратить для создания при фазовом переходе сферического зародыша радиуса $a$ [2], приходим к выражению

$$
R_{\min }=-\frac{8 \pi a^{3} \alpha}{3 a_{\mathrm{c}}}+4 \pi a^{2} \alpha
$$

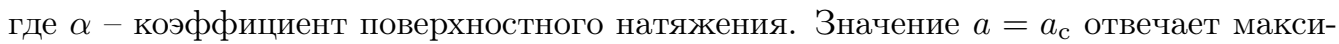
муму функции $R_{\min }(a)$. Вблизи данного максимума

$$
R_{\min }=\frac{4 \pi}{3} \alpha a_{\mathrm{c}}^{2}-4 \pi \alpha\left(a-a_{\mathrm{c}}\right)^{2} .
$$

В итоге приходим к распределению (15). Оно может быть выведено из (12) непосредственно, если выбрать коэффициент $A(a)$ в виде $A(a)=-A_{0} /\left(a-a_{\mathrm{c}}\right)$ и пренебречь более медленной логарифмической зависимостью после интегрирования. При этом возникновение зародышей различного размера рассматривается в среде, которая при этом считается равновесной. Но поскольку состояние метастабильной фазы не отвечает полному статистическому равновесию, то такое рассмотрение относится к временам, малым по сравнению с временем (обратной вероятности) образования критических зародышей, за которым следует переход в новую фазу $\left(a<a_{\mathrm{c}}\right)[2]$. В случае сложных, неаддитивных систем функция $f_{0}(a)$ может иметь вид распределения Тсаллиса [14], которое определяется через деформированную экспоненту $\exp _{q}(x) \equiv[1+(1-q) x]_{+}^{1 /(1-q)},[y]_{+}=\max (y, 0)$ :

$$
f_{0}(a)=C_{1}\left[C_{2}\left(a-a_{\mathrm{c}}\right)^{2}+1\right]^{1 /(1-q)},
$$

где $C_{1}-$ константа, $C_{2}=(1-q) 4 \pi \sigma / T, q-$ показатель неаддитивности. В пределе $q \rightarrow 1$ данное распределение переходит в (15).

Распределение (19) может быть получено из (12) в приближении факторизации. Для этого коэффициент $A(a)$ выберем в виде $A(a)=1 /\left(C_{2}\left(a-a_{\mathrm{c}}\right)^{2}+1\right), \alpha=1, \lambda=0$, коэффициентом $B(a)$ пренебрежем (что является допустимым на микроскопическом уровне, поскольку распределение соответствует обратимым процессам до стадии возникновения фазовых переходов). Получим

$$
\varphi(a)=C \ln \left(C_{2}\left(a-a_{\mathrm{c}}\right)^{2}+1\right)+C_{3},
$$

$C_{3}$ - константа. Полагая $C=1 /(1-q)$, получим функцию $f(a)$ в виде $(19)\left(C_{1}=e^{C_{3}}\right)$. Данное распределение также рассматривается на временах, малых по сравнению с временем образования критических зародышей. 
Равновесное распределение может иметь вид распределения Леви [15] (которое также является однм из возможных решений дробно-дифференциального уравнения Фоккера-Планка [3]) или какой-либо другой соответствующий вид.

Общее условие для равновесного состояния дробно-дифференциального кинетического уравнения (6) с учетом свойств производной Капуто имеет вид

$$
{ }^{c} D_{t}^{\gamma} f(t, a)=0
$$

или

$$
D_{b+}^{\alpha}[A(a) f(t, a)]+D_{b+}^{\alpha_{1}}[B(a) f(t, a)]=0 .
$$

Отсюда с учетом свойств дробного дифференцирования и интегрирования следует связь между коэффициентами $A(a)$ и $B(a)\left(\alpha_{1}=2 \alpha\right)$

$$
B(a)=-\frac{1}{f_{0}} I_{a}^{\alpha} A(a) f_{0}(a)
$$

где

$$
I_{a}^{\alpha} F(a)=\frac{1}{\Gamma(\alpha)} \int_{0}^{y} F(a)(y-a)^{\alpha-1} d a
$$

- дробный интеграл Римана-Лиувилля порядка $\alpha$ от некоторой функции $F(a)$.

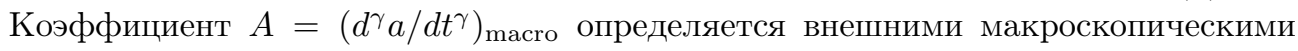
параметрами, в частности концентрацией вещества. Для слабых растворов концентрация $c_{0}$ насышенного раствора над сферической поверхностью радиуса $a$ растворяемого вещества определяется как [2]

$$
c_{0}=c_{\infty}+\frac{a_{\mathrm{c}}}{a}\left(c-c_{\infty}\right)
$$

где $a_{\mathrm{c}}=2 \alpha v c_{\infty} / T\left(c-c_{\infty}\right)$ - критический радиус, $v$ - молекулярный объем вещества зародыша, $c_{\infty}-$ концентрация насыщенного раствора над плоской поверхностью растворяемого вещества.

Подвод вещества к растущему надкритическому зародышу осуществляется диффузией из окружающего раствора. В стационарном режиме сферически-симметричное распределение концентрации $c(r)$ вокруг зародыша радиуса $a$ в среде, обладающей определенными особенностями, в частности фрактальностью, определяется решением дробного диффузного уравнения с лапласианом дробного порядка

$$
D(-\Delta)^{2-\alpha} c(r)=0
$$

где $D-$ коэффициент диффузии, $(-\Delta)^{2-\alpha} c(r)=-\Delta I_{\mathrm{d}}^{\alpha} c(r)$, где $I_{\mathrm{d}}^{\alpha}-$ потенциал Рисса [16], $\Delta=(1 / r)\left(\partial^{2} / \partial r^{2}\right) r$. Поскольку рассматривается сферически-симметричный случай с одной переменной, вместо потенциала Рисса будем использовать дробную производную Капуто с конечными пределами. Граничные условия для уравнения (25) имеют вид $c(\infty)=c, c(a)=c_{0}$.

Решение уравнения (25) с учетом граничных условий имеет вид

$$
c(r)=\frac{c_{0} a^{1+\alpha}}{r^{1+\alpha}} .
$$


Дробный диффузный поток по направлению к зародышу

$$
S^{\gamma}=\left.4 \pi r^{2} D \frac{\partial^{\alpha} c}{\partial r^{\alpha}}\right|_{r=a}=\frac{4 \pi D B_{z}(-\alpha, 1+2 \alpha) \alpha}{\Gamma(1-\alpha)}\left(c_{\infty} a^{2-\alpha}+a_{\mathrm{c}} a^{1-\alpha}\left(c-c_{\infty}\right)\right),
$$

где $B_{z}$ - неполная бета-функция [17].

Таким образом, имеем

$$
A(a)=\left(\frac{d^{\gamma} a}{d t^{\gamma}}\right)_{\text {macro }}=\frac{S^{\gamma} v}{4 \pi a^{2}}=\frac{4 \pi D B_{z}(-\alpha, 1+2 \alpha) \alpha}{\Gamma(1-\alpha)}\left(\frac{c_{\infty}}{a^{\alpha}}+\frac{a_{\mathrm{c}}\left(c-c_{\infty}\right)}{a^{1+\alpha}}\right) .
$$

В соответствии с (22) для равновесной функции вида (15), интегрируя в пределах от 0 до $a_{\mathrm{c}}$, получим функцию $B(a)$ :

$$
\begin{aligned}
B=\frac{4 \pi D B_{z}(-\alpha, 1+2 \alpha) \Gamma(\alpha) \alpha}{f_{0}(a) \Gamma(1-\alpha)}\left(c_{\infty 2} F_{2}\left(\frac{\alpha}{2} ; \frac{\alpha+1}{2} ; \frac{1}{2} ; 1 ; \sigma a_{\mathrm{c}}^{2}\right)+\right. \\
\left.+\frac{\left(c-c_{\infty}\right) e^{\alpha \pi i}}{4 \pi \sin (-\alpha \pi)}{ }_{2} F_{2}\left(\frac{\alpha}{2} ; \frac{\alpha+1}{2} ; 0 ; \frac{1}{2} ; \sigma a_{\mathrm{c}}^{2}\right)\right)
\end{aligned}
$$

где $\sigma=4 \pi \lambda /\left(k_{\mathrm{B}} T\right),{ }_{2} F_{2}$ - обобщенный гипергеометрический ряд [18]. Отметим, что для второго слагаемого интегрирование производилось по петле Похгаммера [16].

Используя (19), (22) и (28), получим

$$
\begin{aligned}
& B(a)=\frac{4 \pi D B_{z}(-\alpha, 1+2 \alpha) \Gamma^{2}(\alpha) \alpha C_{1}}{C_{2}^{1-q} f_{0}(a)} \frac{\left(\sqrt{C_{2}}\right)^{2 /(1-q)}}{\Gamma(1-\alpha)} \times \\
& \times\left(c_{\infty 3} F_{2}\left(-\frac{1}{1-q} ; \frac{\alpha}{2} ; \frac{\alpha+1}{2} ; \frac{1}{2} ; 1 ;-\frac{a_{\mathrm{c}}^{2}}{C_{2}}\right)+\right. \\
& \left.+\frac{\left(c-c_{\infty}\right) e^{\alpha \pi i}}{4 \pi \sin (-\alpha \pi)}{ }_{3} F_{2}\left(-\frac{1}{1-q} ; \frac{\alpha}{2} ; \frac{\alpha+1}{2} ; 0 ; \frac{1}{2} ;-\frac{a_{\mathrm{c}}^{2}}{C_{2}}\right)\right) \text {, }
\end{aligned}
$$

где ${ }_{3} F_{2}$ - обобщенный гипергеометрический ряд [18]. Ряд ${ }_{2} F_{2}$ является сходящимся. Чтобы сходился ряд ${ }_{3} F_{2}$, необходимо выполнение условия $\left|a_{\mathrm{c}}^{2} / C_{2}\right|<1$.

Рассмотрим общее стационарное решение уравнения (7)

$$
f(a)=C \exp \left[-\int \frac{\lambda+\tilde{A}^{\alpha}(a)+\widetilde{B}^{\alpha_{1}}(a)}{\tilde{A}^{\alpha-1}(a)+\widetilde{B}^{\alpha_{1}-1}(a)} d a\right]
$$

где $C$ - константа. (Распределения (15), (19) могут быть получены непосредственно из (31).)

Граничные условия при $a \rightarrow 0$ можно выбрать в виде $f / f_{0} \rightarrow 1$, а при $a \rightarrow \infty-$ в виде $f / f_{0}=0$.

Покажем, что дробно-дифференциальное уравнение Фоккера-Планка действительно описывает фазовые переходы. В силу универсальности фазовых переходов и для общности результатов рассмотрим следующее решение.

Выберем коэффицент дрейфа в виде

$$
A(a)=\frac{1}{T\left(4 B_{0} a^{3}+2 a_{0} t-h\right)} .
$$




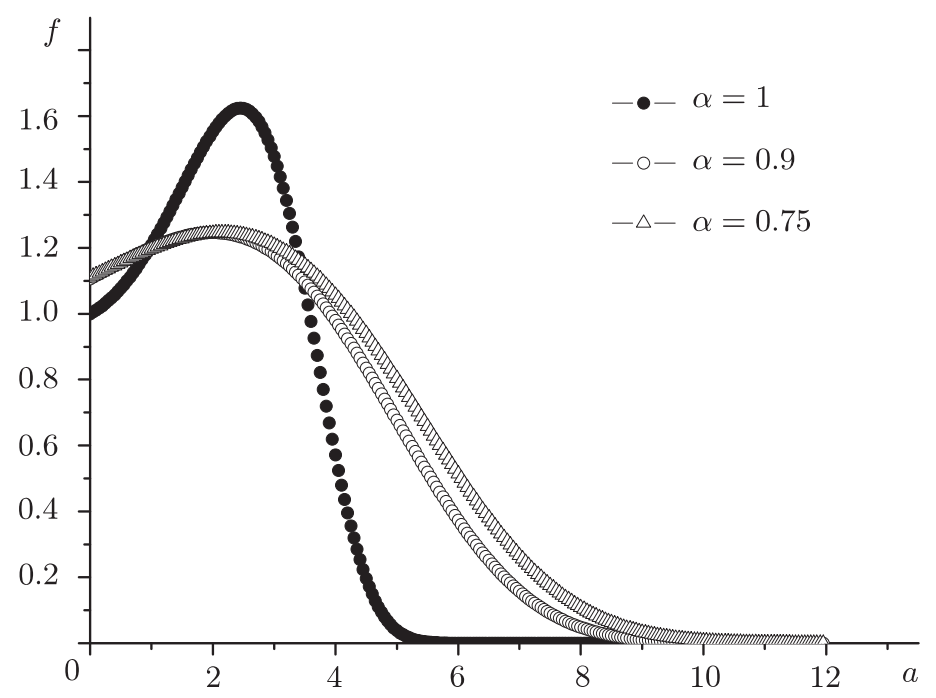

Рис. 2. Графики функций распределения в точке фазового перехода для различных $\alpha$.

Подставим (32) в (31) при $\alpha=1$, пренебрежем коэффициентом диффузии $B$, а также производной $A^{\alpha}$ в числителе, получим решение в виде

$$
f(a)=f_{1} e^{-F / T}
$$

где $F$ - свободная энергия,

$$
F=B_{0} \lambda a^{4}+a_{0} \lambda t a^{2}-h \lambda a,
$$

где $t=\left(T-T_{\mathrm{c}}\right) / T_{\mathrm{c}}-$ разность температур, $T_{\mathrm{c}}$ - критическая температура фазового перехода, $B_{0}, a_{0}, \lambda, f_{1}$ - константы, $h$ - внешнее поле. Здесь мы пренебрегли слагаемыми с более медленной зависимостью от $a$. Зависимость (34) для значений $t=-0.1, a_{0}=1, B_{0}=0.01, f_{1}=1, \lambda=1, h=0.1$ показана на рис. 2. В данной системе единиц температура измеряется в единицах критической температуры $\left(T_{\mathrm{c}}=1\right)$, параметр порядка $a$ в единицах $a_{\mathrm{c}}\left(a_{\mathrm{c}}=1\right)$, индукция магнитного поля $h$, коэффициенты $B_{0}, a_{0}$ в единицах $k_{\mathrm{B}} T_{\mathrm{c}}, \lambda$ - безразмерный параметр, $\lambda=1$.

Если при заданной температуре $t<0$ менять поле, то при прохождении им нулевого значения возникает фазовый переход первого рода. На рис. 3 приведена зависимость параметра порядка $a$ от внешнего поля. Прерывистой линией отмечена область метастабильных состояний. Таким образом, при $\alpha=1$ уравнение (6) действительно описывает фазовые переходы первого рода.

Рассмотрим случай дробных $\alpha$. Выберем $A(a)$ также в виде (32). Подставим (32) в (31) при $\alpha=0.75$, пренебрежем коэффициентом диффузии $B$, а также производной $A^{\alpha}$ в числителе, получим решение в виде $(34)$, где

$$
F=F_{1} a^{15 / 4}+F_{2} t a^{7 / 4}-h F_{3} a^{3 / 4}
$$




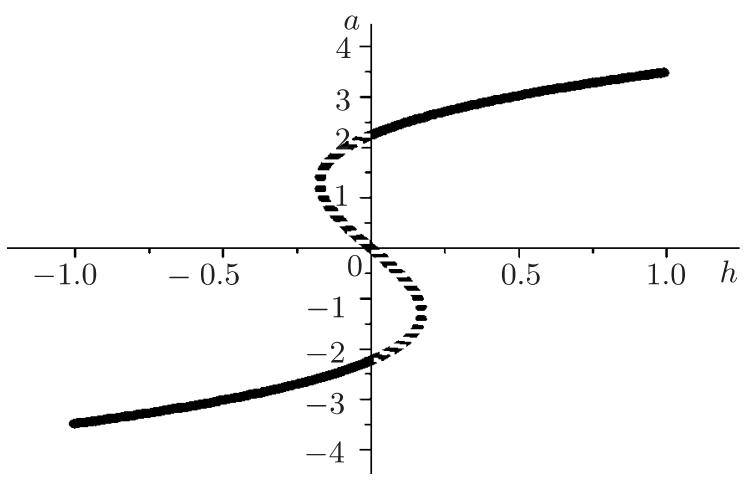

Рис. 3. График зависимости параметра порядка от внешнего поля $(\alpha=1)$.

где

$$
\begin{aligned}
& F_{1}=\Gamma(2-\alpha)\left(1-3.2 \frac{\Gamma(2-\alpha) \Gamma(\alpha)}{\Gamma(\alpha-1) \Gamma(3-\alpha)}\right) \frac{\lambda B_{0}}{x_{0}^{2}}, \\
& F_{2}=\Gamma(2-\alpha)\left(1.14-5.7 \frac{\Gamma(2+\alpha) \Gamma(\alpha)}{\Gamma(\alpha-1) \Gamma(3-\alpha)}\right) \frac{\lambda a_{0}}{x_{0}^{2}}, \\
& F_{3}=\Gamma(2-\alpha)\left(1.33-8 \frac{\Gamma(2+\alpha) \Gamma(\alpha)}{\Gamma(\alpha-1) \Gamma(3-\alpha)}\right) \frac{\lambda a_{0}}{x_{0}^{2}}, \\
& x_{0}=-1+3 \frac{\Gamma(2-\alpha) \Gamma(\alpha)}{\Gamma(\alpha-1) \Gamma(3-\alpha)} .
\end{aligned}
$$

Здесь мы также принебрегли слагаемыми с более медленной зависимостью от $a$. Данное решение показано на рис. 2. Видно, что для дробного порядка решение спадает более плавным образом, как бы размывается. Вершина пика также расположена ниже, чем при $\alpha=1$. Зависимость параметра порядка $a$ от внешнего поля практически идентична приведенной на рис. 3 , и соответственно фазовый переход имеет место в данной системе. Отличие проявляется в увеличении ширины критической области для индекса $\alpha$ нецелого порядка.

Для $\alpha=0.9$ получим

$$
F=F_{1} a^{39 / 10}+F_{2} t a^{19 / 10}-h F_{3} a^{9 / 10},
$$

где

$$
\begin{aligned}
& F_{1}=\Gamma(2-\alpha)\left(1-3.077 \frac{\Gamma(2-\alpha) \Gamma(\alpha)}{\Gamma(\alpha-1) \Gamma(3-\alpha)}\right) \frac{\lambda B_{0}}{x_{0}^{2}}, \\
& F_{2}=\Gamma(2-\alpha)\left(1.05-5.26 \frac{\Gamma(2+\alpha) \Gamma(\alpha)}{\Gamma(\alpha-1) \Gamma(3-\alpha)}\right) \frac{\lambda a_{0}}{x_{0}^{2}}, \\
& F_{3}=\Gamma(2-\alpha)\left(1.11-6.67 \frac{\Gamma(2+\alpha) \Gamma(\alpha)}{\Gamma(\alpha-1) \Gamma(3-\alpha)}\right) \frac{\lambda a_{0}}{x_{0}^{2}} .
\end{aligned}
$$

Решение для $\alpha=0.9$ показано на рис. 2. Видно, что с уменьшением $\alpha$ решения спадают более плавным образом, пик расширяется. При $\alpha=0.9$ зависимость параметра порядка $a$ от внешнего поля аналогична вышеупомянутым зависимостям для 
$\alpha=0.75,1$. С уменьшением $\alpha$ происходит расширение критической области. Так, для $\alpha=1$ величина критического поля [19], определяющая границы интервала, на котором возникают три вещественных корня уравнения параметра порядка (рис. 3), при указанных выше параметрах составляет $h_{\mathrm{t}}=0.172$, для $\alpha=0.9-h_{\mathrm{t}}=0.178$, а для $\alpha=0.75-h_{\mathrm{t}}=0.182$.

Таким образом, дробно-дифференциальное уравнение типа Фоккера-Планка действительно описывает фазовые переходы.

\section{4. ЗАКЛЮЧЕНИЕ}

При фазовых переходах, в частности при описании роста зародышей во фрактальной среде, наблюдается конкуренция двух странных процессов. С одной стороны, это так называемые случайные блуждания во фрактальном времени или, точнее, случайные вариации размеров, связанные с фрактальным временем. Данные процессы являются немарковскими и обладают памятью среды: последующие вариации размеров связаны с предыдущими изменениями. С другой стороны, имеют место процессы, описываемые пространственной дробной производной, - так называемые процессы Леви, связанные с нелокальностью. С теоретической точки зрения это подразумевает мгновенные скачки размеров зародышей. Данные скачки размеров должны возникать с большей вероятностью, чем изменения, связанные с фрактальным временем, что может привести к расходимости среднеквадратичного изменения размеров зародышей (об устранении расходимости см. [20]).

Конкуренция между двумя вышеупомянутыми процессами может привести к сложной структуре изменения размеров частиц. K примеру, размер зародыша может долгое время не меняться, а затем проявить резкую активность, в соответствии с картиной так называемого перемежаемого типа движения.

Кроме того, в работе показано, что при фазовых переходах во фрактальной среде может иметь место деформация статистики, связанная с проявлением негауссова распределения и эффектов неаддитивности.

\section{Список литературы}

[1] Я. Б. Зельдович, ЖЭТФ, 12 (1942), 525-538.

[2] Е. М. Лифшиц, Л. П. Питаевский, Теоретическая физика, т. 10: Физическая кинетика, Физматлит, М., 2002.

[3] V.E. Tarasov, Fractional Dynamics: Applycation of Fractional Calculus to Dynamics of Particles, Fields and Media, Springer, Heidelberg; Higher Education Press, Beijing, 2010.

[4] Г. И. Капель, С.В. Разоренов, А.В. Уткин, В.Е. Фортов, Ударно-волновые явления в конденсированных средах, Янус-К, М., 1996.

[5] Л. Пьетронеро, Э. Тозатти (ред.), Фракталь в физике, Труды VI Международного симпозиума по фракталам в физике (МЦТФ, Триест, Италия, 9-12 июля 1985), Мир, M., 1988.

[6] A. V. Milovanov, L. M. Zelenyi, G. Zimbardo, P. Veltri, J. Geophys. Res., 106:A4 (2001), 6291-6307.

[7] K. Rypdal, J.-V. Paulsen, O. E. Garcia, S. V. Ratynskaia, V. I. Demidov, Nonlin. Processes Geophys., 10 (2003), 139-149.

[8] A. V. Milovanov, J. J. Rasmussen, Phys. Rev. B, 66:13 (2002), 134505, 11 pp.

[9] А.И. Олемской, Синергетика сложных систем. Феноменология и статистическая теория, Эдиториал УРСС, М., 2009. 
[10] G. M. Zaslavsky, "Fractional kinetics of Hamiltonian chaotic systems", Application of Fractional Calculus in Physics, ed. R. Hilfer, World Sci., River Edge, NJ, 2000, 203-239.

[11] I. Podlubny, Fractional Differential Equations. An Introduction to Fractional Derivatives, Fractional Differential Equations, to Methods of their Solution and Some of their Applications, Mathematics in Science and Engineering, 198, Academic Press, San Diego, CA, 1999.

[12] K. B. Oldham, J. Spanier, The Fractional Calculus. Theory and Applications of Differentiation and Integration to Arbitrary Order, Mathematics in Science and Engineering, 111, Academic Press, New York, London, 1974.

[13] A. M. Mathai, R. K. Saxena, H. J. Haubold, The H-Function. Theory and Application, Springer, Dordrecht, 2010.

[14] K. Tsallis, Introduction in Nonextensive Statistical Mechanics. Approaching a Complex World, Springer, Berlin, 2009.

[15] Б. Б. Мандельброт, Фрактальная геометрия природы, ИКИ, М., 2002.

[16] С. Г. Самко, А. А. Килбас, О. И. Маричев, Интеграль и производные дробного порядка и некоторые их применения, Наука и техника, Минск, 1987.

[17] А. П. Прудников, Ю. А. Брычков, О.И. Маричев, Интегралы и рлды, т. 3: Специалъные функиии. Дополнительные главы, Физматлит, М., 2003.

[18] H. Bateman, Higher Transcedental Function, v. 1, McGraw-Hill, New York, 1953.

[19] Л. Д. Ландау, Е. М. Лифшиц, Теоретическая физика, т. 5: Статистическая физика, Физматлит, М., 2002.

[20] Л. М. Зелёный, А. В. Милованов, УФН, 174:8 (2004), 809-852.

Поступила в редакцию 2.12.2012, после доработки 7.04.2013 